\title{
Innovative Mapping of Mineral and Organic Matter of Source Rocks at the Nanometer Scale Using Back Scatter Electron and Energy Dispersive Spectroscopy
}

\author{
David Jacobi ${ }^{1}$, Qiushi Sun ${ }^{1}$, John Longo ${ }^{1}$, Josh Brothers ${ }^{1}$ and Jordan Kone ${ }^{2}$ \\ 1. Aramco Services, Houston Research Center, Reservoir Engineering, Houston, Texas, USA. \\ 2. Carl Zeiss, ZEISS Microscopy Customer Center, Pleasanton, California, USA.
}

Successful exploitation of hydrocarbons from a source rock reservoir requires knowledge of the organic matter type, its concentration, and its fabric throughout the mineral matrix. However, quantifying and mapping these rock features at different length scales can be difficult. As an example, current advances in the use of electron dispersive spectroscopy (EDS) and back scatter electron (BSE) data acquired using two-dimensional scanning electron microscopy (2D-SEM) have thus far been inadequate in resolving features present in source rock images because the spatial resolution is typically $1 \mu \mathrm{m}$.

A spatial resolution of $1 \mu \mathrm{m}$ is usually sufficient at effectively quantifying and mapping the pervasive, coarse grain mineralogy of most sedimentary rock reservoirs, such as those of sandstones and limestone. The mineral matrices of source rocks, consisting of fine grained, organic shales however, are more complex and heterogeneous. Laminated, layered rock fabric can vary in thicknesses on the order of nanometers, and the concentrations and distributions of mineral phases and organic matter can change within $250 \mu \mathrm{m}$ (Fig.1a). In comparison, $250 \mu \mathrm{m}$ is the diameter of a single quartz grain in a sandstone reservoir. Mineral maps of source rocks produced with a spatial resolution of $1 \mu \mathrm{m}$ are usually granular and highly pixelated. The maps do not distinctly resolve the boundaries and geometries of the smaller grain sizes found in the matrix.

To address this challenge, a new method is presented that utilizes an integrated rules based vector algorithm in conjunction with the relative intensities of EDS and BSE data to map the mineralogy and organic matter from 2D-SEM images with nanometer resolution (Fig. 1b). In addition to resolving the grain boundaries and morphology of the complex mineral matrices of source rocks, the maps can also resolve, on a nanometer scale, the finely disseminated grains of organic matter that contribute to hydrocarbon production.

The limited resolution of mapping software is often explained as the result of the $0.75 \mu \mathrm{m}$ diameter spot size of the electron beam used to acquire the EDS and BSE data. The acquired intensities are empirically converted to element concentrations and " $Z$ " values [1] to quantify mineralogy using least squares minimization algorithms. The map resolution obtained from these algorithms using EDS and BSE are constrained by the beam size, making it difficult for least squares algorithms to resolve compositional collinearity among minerals or differences in inorganic versus organic carbon between carbonate minerals versus organic matter (Fig.1c) As a result, many software applications are unable to differentiate between mineral phases of similar composition and make no attempt to quantify or map the organic matter in source rocks.

The new software initially was developed using a cellular spreadsheet program where each pixel, containing intensities from EDS and BSE, defined the boundaries and morphology of the image (Fig. 2a). An expert system then uses relative threshold values to identify, quantify, and map the minerals and organic matter within the image (Fig. 2a). The algorithm can quantify 20 different mineral phases using 
EDS, including organic matter defined by both EDS and BSE data. The rules based expert system was then integrated with a compositional vector based algorithm. The integrated algorithm, compiles and stacks EDS and BSE maps to define a multidimensional array. This array is organized by three indices such that each pixel of the image represents an elemental vector containing all values of the EDS elements and BSE intensity associated with that pixel (Fig.2b). From these vectors, a weighted mineral variable is produced through a routine of summation and subtraction of element components determined by the mineral definition. This weighted mineral variable, guided by the rules based expert system, then determines the most probable mineral and organic matter per pixel, creating a high-resolution map of the rock surface. This method has also been extended for using micro-X-ray Fluorescence ( $\mu \mathrm{XRF}$ ) data to quantify mineralogy from whole core for upscaling to reservoir dimensions.

References:

[1] GE Lloyd, Mineralogical Magazine 51 (1987), p. 3.
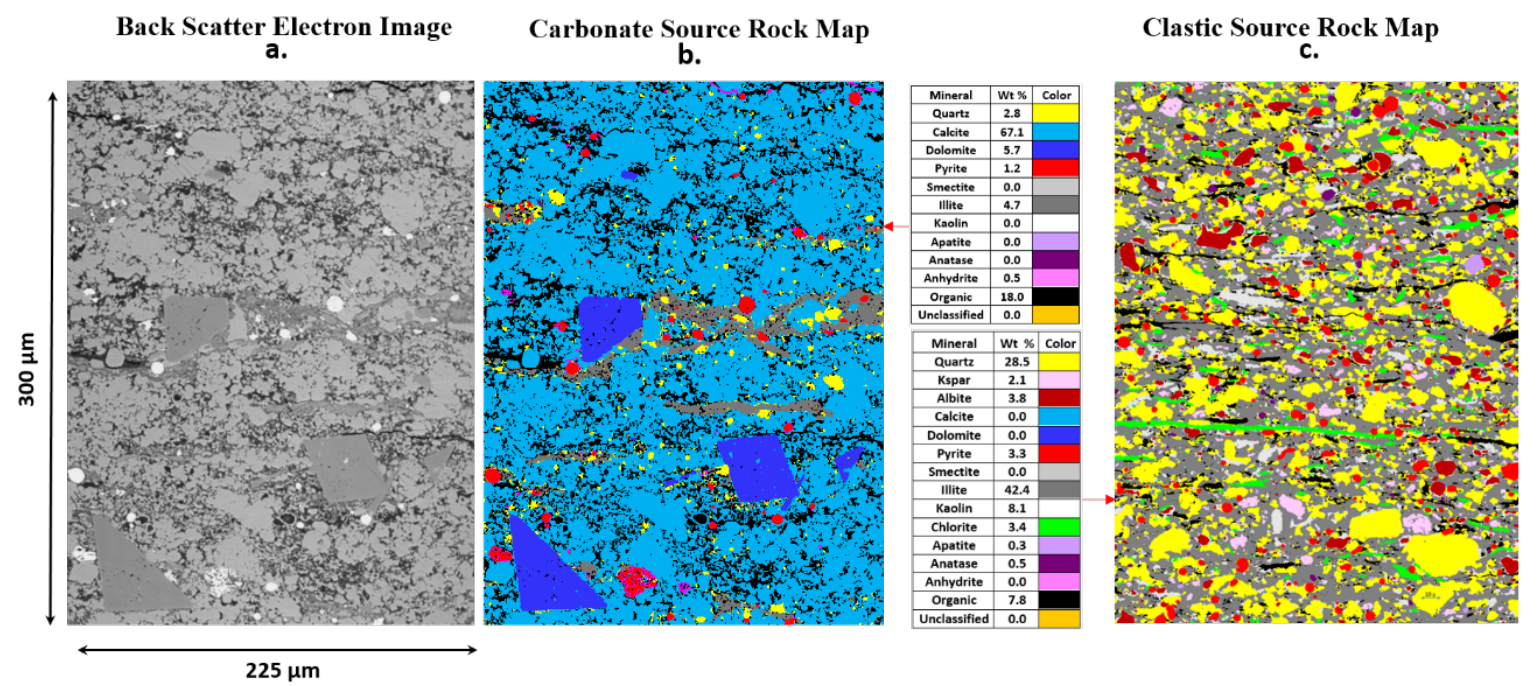

Figure 1. (a.) Back Scatter Electron image showing the heterogeneity of the source rock mineral (grey to white) and organic matter (black) fabric. (b.) Carbonate Source Rock Map showing the ability of the software to distinguish between carbonate, organic matter, and clay minerals along with pyrite framboids. (c.) Clastic Source Rock Map showing different clay type, quartz and feldspar type and organic matter.

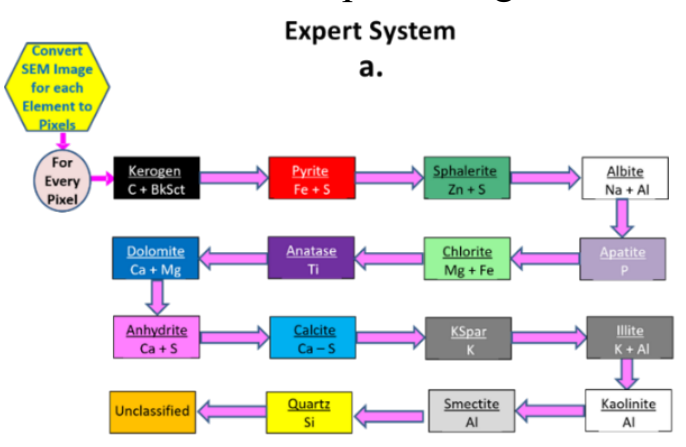

Elemental Map Vectors

b.

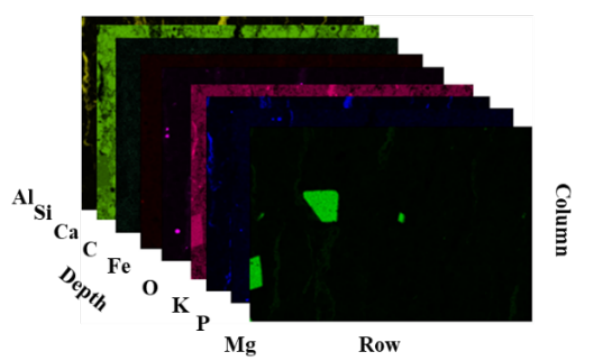

Figure 2. Algorithm Components (a.) Flow diagram for expert system (b.) Multidimensional element array 